\title{
The added value of hybrid ventilation/perfusion SPECT/CT in patients with stable COPD or apparently healthy smokers. Cancer-suspected CT findings in the lungs are common when hybrid imaging is used
}

This article was published in the following Dove Press journal: International Journal of COPD 18 December 2014

Number of times this article has been viewed

\author{
Jonas Jögi' \\ Hanna Markstad ${ }^{2}$ \\ Ellen Tufvesson ${ }^{3}$ \\ Leif Bjermer ${ }^{3}$ \\ Marika Bajc' \\ 'Department of Clinical Physiology \\ and Nuclear Medicine, ${ }^{2}$ Department \\ of Radiology, ${ }^{3}$ Department of \\ Respiratory Medicine and Allergology, \\ Skåne University Hospital and Lund \\ University, Lund, Sweden
}

\begin{abstract}
Ventilation/perfusion (V/P) single-photon emission computed tomography (SPECT) is recognized as a diagnostic method with potential beyond the diagnosis of pulmonary embolism. V/P SPECT identifies functional impairment in diseases such as heart failure (HF), pneumonia, and chronic obstructive pulmonary disease (COPD). The development of hybrid SPECT/computed tomography (CT) systems, combining functional with morphological imaging through the addition of low-dose CT (LDCT), may be useful in COPD, as these patients are prone to lung cancer and other comorbidities. The aim of this study was to investigate the added value of LDCT among healthy smokers and patients with stable COPD, when examined with V/P SPECT/CT hybrid imaging. Sixty-nine subjects, 55 with COPD (GOLD I-IV) and 14 apparently healthy smokers, were examined with V/P SPECT and LDCT hybrid imaging. Spirometry was used to verify COPD grade. Only one apparently healthy smoker and three COPD patients had a normal or nearly normal V/P SPECT. All other patients showed various degrees of airway obstruction, even when spirometry was normal. The same interpretation was reached on both modalities in 39\% of the patients. LDCT made V/P SPECT interpretation more certain in $9 \%$ of the patients and, in 52\%, LDCT provided additional diagnoses. LDCT better characterized the type of emphysema in 12 patients. In 19 cases, tumor-suspected changes were reported. Three of these 19 patients (ie, $4.3 \%$ of all subjects) were in the end confirmed to have lung cancer. The majority of LDCT findings were not regarded as clinically significant. V/P SPECT identified perfusion patterns consistent with decompensated left ventricular HF in 14 COPD patients. In 16 patients (23\%), perfusion defects were observed. HF and perfusion defects were not recognized with LDCT. In COPD patients and long-time smokers, hybrid imaging had added value compared to V/P SPECT alone, by identifying patients with lung malignancy and more clearly identifying emphysema. V/P SPECT visualizes comorbidities to COPD not seen with LDCT, such as pulmonary embolism and left ventricular HF.
\end{abstract}

Keywords: lung, lung cancer, V/P SPECT, ventilation/perfusion, single-photon emission computed tomography, computed tomography

\section{Background}

Chronic obstructive pulmonary disease (COPD) is a heterogeneous disease, and patients with COPD are prone to comorbid diseases such as heart failure, ischemic heart disease, and lung malignancy. ${ }^{1}$ Ventilation/perfusion (V/P) single-photon emission computed
Correspondence: Marika Bajc Department of Clinical Physiology and Hospital and Lund University, S22I 85 Lund, Sweden

Tel +46 46173303

Email marika.bajc@med.lu.se 
tomography (SPECT) is a nuclear medicine method that is used primarily for pulmonary embolism (PE) diagnosis, but which has also been shown to be a sensitive method by which to detect and stage the degree of airway obstruction and other parenchymal changes. ${ }^{2,3}$ SPECT/computed tomography (CT) hybrid systems are becoming commonly used. The hybrid SPECT/CT system is a dual-imaging modality technique whose clinical application has, so far, had the most impact in oncological diseases as a result of improved sensitivity and specificity, and because it combines co-registration of anatomical and functional data. ${ }^{4,5}$ This system can improve the staging and monitoring of treatment. As nuclear medicine procedures have the ability to visualize functional changes before structural changes occur, the addition of a CT procedure can allow morphological correlation of regions with physiological variants. It can also be used for correction for photon attenuation. Regarding the lungs, low-dose CT (LDCT) has been discussed as a screening method for lung cancer among elderly persons with an elevated risk, eg, smokers, but guidelines for if and whom to screen have not yet been established. ${ }^{6}$ In V/P SPECT, co-registered CT has been used by some authors to improve the specificity in $\mathrm{PE}$ diagnosis, but the value of adding LDCT has otherwise been insufficiently studied. ${ }^{7}$

The objective of this study was therefore to investigate what the added value of LDCT was among healthy smokers and patients with stable COPD when examined with V/P SPECT/CT hybrid imaging.

\section{Subjects and methods}

This prospective study was performed with approval from the Regional Ethical Committee in Lund, Sweden. All 69 subjects gave their informed consent of participation. Patients who were either apparently healthy current or former longtime smokers $(n=14$; mean age \pm standard deviation $=69 \pm 3$ years; seven women, smoking 24-40 pack-years) or patients with stable COPD $(n=55$; mean age \pm standard deviation $=68 \pm 5$ years; 25 women; Global initiative for chronic Obstructive Lung Disease [GOLD] stage I-IV, smoking 15-159 pack-years), were examined with V/P SPECT and LDCT in a hybrid system. To be included in the study subjects had to be over the age of 40 years and clinically stable and, in the case of COPD patients, had been without any exacerbations during the 4 weeks prior to inclusion. Patients that did not fulfill the inclusion criteria were excluded from the study. Spirometry was performed in accordance with the guidelines of the American Thoracic Society to measure the ventilatory impairment and validate the GOLD stage. ${ }^{8}$ The
V/P SPECT images were first interpreted independently of the CT examination by an experienced nuclear physician. The assessment was done in accordance with the guidelines of the European Association of Nuclear Medicine (EANM). ${ }^{9}$ Then, the V/P SPECT images were reinterpreted in combination with the CT images. This was done together with an experienced chest radiologist. V/P SPECT/CT fusion images were also available at this time. It was noted whether the interpretation of the examination was the same as the one made without use of LDCT, if LDCT helped to make the interpretation more definite, or if LDCT added new diagnoses not observed with V/P SPECT. The physicians who reviewed the V/P SPECT and LDCT images were blinded to all other information. Suspected tumor changes in LDCT were reported and follow-up was performed by referring physicians in accordance with local guidelines of Skåne University Hospital, Lund, Sweden.

\section{V/P SPECT/CT protocol}

All examinations were performed with a Precedence SPECT/ CT system (Philips Healthcare, Best, the Netherlands), which combines a dual-head gamma camera with a Brilliance 16-slice CT scanner. The procedure was begun with a CT overview image and continued with diagnostic LDCT (120 $\mathrm{kV}, 20 \mathrm{mAs} / \mathrm{slice}, 16 \times 1.5$ collimator, 0.5 second rotation time, and pitch of 0.813 ). The CT examination was not used for attenuation correction, but to colocalize the morphological and functional changes visualized in either of the two modalities. The CT examination was performed during tidal breathing. The CT acquisition was followed by the protocol for V/P SPECT, which has been described earlier ${ }^{2}$ and which is in accordance with the guidelines of EANM. ${ }^{9}$

For the V/P SPECT, administration of ventilation and perfusion agents was performed in the supine position so as to minimize gravitational gradients. The V/P SPECT procedure starts with ventilation scintigraphy upon inhalation of very fine ${ }^{99 \mathrm{~m}}$ Tc-labeled solid graphite particles (Technegas; Cyclomedica, Lucas Heights, NSW, Australia), which are mainly deposited in the alveoli by diffusion. Doses of 25-30 MBq in the lungs are needed for ventilation studies. Perfusion tomography follows immediately after ventilation, without changing the position of the patient. For perfusion scintigraphy, $120 \mathrm{MBq}$ of technetium-labeled particles of macroaggregates of human albumin ( $\left.{ }^{99 \mathrm{~m}} \mathrm{Tc}-\mathrm{MAA}\right)$ are injected intravenously. A general purpose collimator, 64×64 matrix, is used. The total number of projections is 128 (64 stops for each of the two gamma camera heads). Each stop is 10 seconds for the ventilation study, and, for the perfusion 
study, 5 seconds. This allows a total acquisition time of 20 minutes. For V/P SPECT reconstruction, ordered-subset expectation maximization iterative reconstruction with eight subsets and two iterations is used.

\section{Results}

\section{V/P SPECT in apparently healthy smokers}

Thirteen of the 14 apparently healthy smokers demonstrated various degrees of airway obstruction on V/P SPECT, although spirometry did not detect decreased ventilation capacity or airflow limitation. Only one smoker had a completely normal V/P distribution.

Five subjects showed perfusion defects in ventilated regions (mismatch). Six apparently healthy smokers showed matched V/P defects typical for emphysema, with extension of $5 \%-25 \%$. One subject showed V/P impairment that is typical for pneumonia.

\section{V/P SPECT in COPD patients}

Three COPD patients had nearly normal ventilation according to V/P SPECT; one patient in GOLD stage I and two patients in GOLD stage II. All of the others showed different degrees of airway obstruction. Emphysema was identified in 48 patients, with extension of $5 \%-80 \%$. In ten of the 55 patients, vascular defects (mismatch) were observed. Moreover, V/P SPECT identified 14 patients with a perfusion pattern consistent with pulmonary congestion typical for decompensated left ventricular HF (Figure 1). ${ }^{10}$

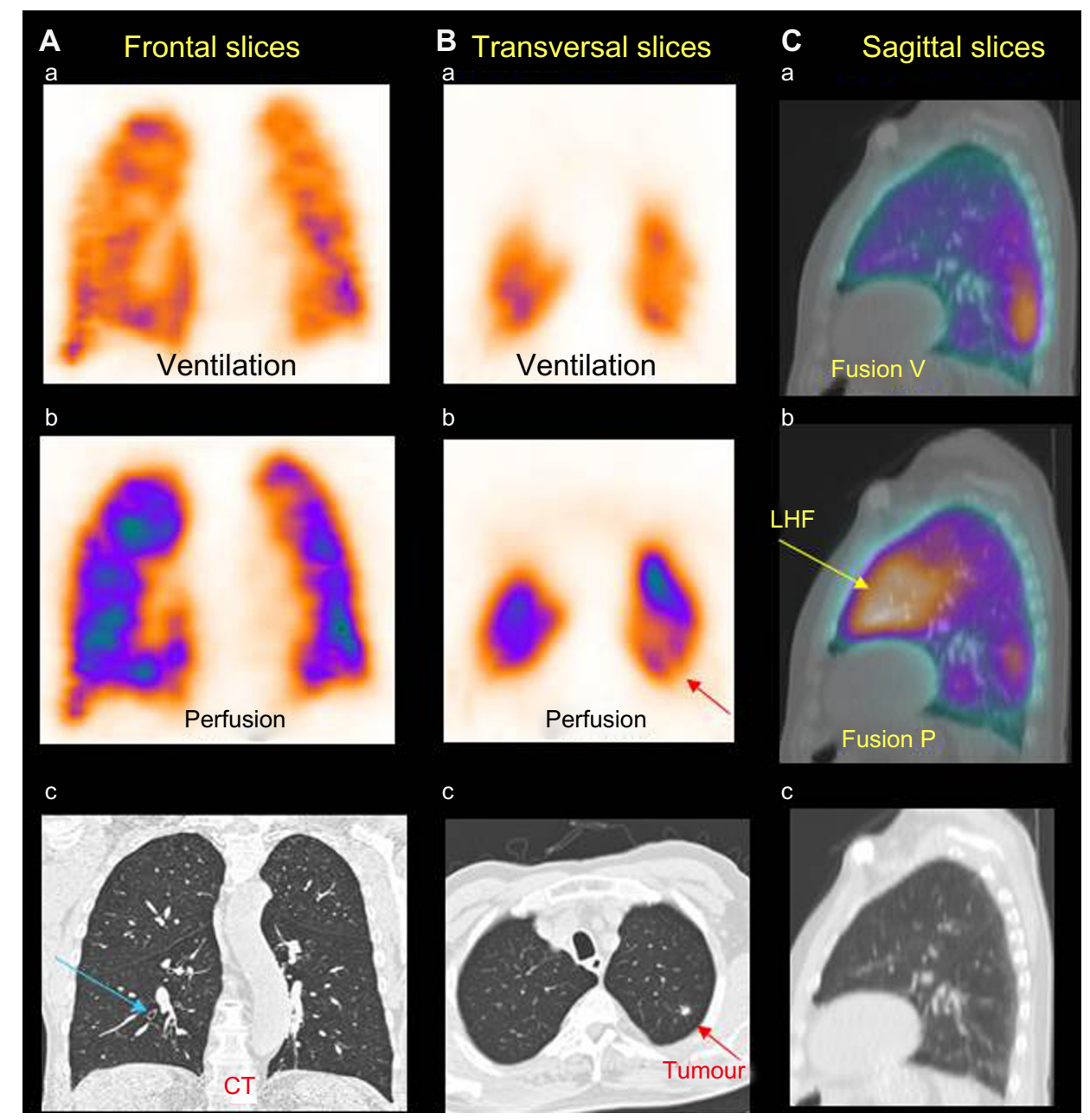

Figure I Patient with COPD, LHF, and a small tumor.

Notes: (A) Frontal slice of ventilation SPECT (a) showing uneven distribution of ventilation with with deposition of Technegas in central airways, which is a typical sign of airway obstruction. Corresponding perfusion SPECT (b) and CT image (c) are shown. The blue arrow in (c) shows thickening of the airway wall that is typical of COPD. (B) Transverse slices of ventilation SPECT (a), with corresponding perfusion SPECT (b). In the CT image (c), a small tumor (I cm; red arrow) is visualized. In the corresponding V/P SPECT image (b) a small perfusion defect is observed (red arrow). (C) Sagittal slice showing fusion ventilation SPECT/CT (a), with corresponding fusion perfusion SPECT/CT image (b) that shows antigravitational redistribution of perfusion, typical for LHF (yellow arrow), which is not visible on CT (c) or ventilation images (nonsegmental mismatch).

Abbreviations: COPD, chronic obstructive pulmonary disease; CT, computed tomography; LHF, left ventricular heart failure; SPECT, single-photon emission computed tomography. 
Table I Added value of LDCT with V/P SPECT versus V/P SPECT alone

\begin{tabular}{lll}
\hline Impact of LDCT & n (69) & $\%$ \\
\hline Same interpretation, nothing new & 27 & 39 \\
Interpretation more clear/certain & 6 & 9 \\
Additional diagnosis that did not change treatment & 27 & 39 \\
Additional diagnosis that led to changed & 9 & 13 \\
or new treatment & &
\end{tabular}

Abbreviations: LDCT, low-dose computed tomography; V/P SPECT, ventilation/ perfusion single-photon emission computed tomography.

\section{Additional value of LDCT}

The same interpretation on both modalities was reached in 27 subjects (39\%). Interpretation of V/P SPECT was more definite in six patients $(9 \%)$. CT provided additional diagnoses in 36 patients (52\%) (Table 1). Table 2 shows the type and distribution of the LDCT findings that were found among the 36 patients with additional diagnoses on LDCT in Table 1. Most of the diagnoses were not regarded as clinically significant. LDCT better characterized the type of emphysema in 12 patients. In 19 cases, nodular or tumorsuspected findings in LDCT images were reported (Table 2). Nine patients (13\%) needed further follow-up and, of these, three patients $(4.3 \%)$ were found to have lung cancer (Figure 2 ). One of the patients died due to this malignancy during the follow-up period.

LDCT did not recognize the decompensation or the vascular defects seen in V/P SPECT images in any case.

\section{Discussion}

This study shows that the strength of hybrid imaging in patients with COPD and long-term smokers is primarily in detecting tumor-suspected changes and lung cancer. COPD patients are known to be prone to tumor development, and screening of selected high-risk patients using LDCT has been recommended by some authors and been shown to reduce lung cancer mortality by $20 \%$ compared with chest

Table 2 Distribution of CT findings among patients with additional diagnoses on LDCT

\begin{tabular}{ll}
\hline LDCT findings & $\mathbf{n}^{\mathbf{a}} \mathbf{( 3 6 )}$ \\
\hline Nodular changes & 19 \\
Pleural effusion (minor) & $\mathrm{I}$ \\
Emphysema (better characterization) & 12 \\
Atelectasis & 2 \\
Bronchiolitis or bronchiectasis & 4 \\
Parenchymal changes & 4 \\
Vascular malformation (minor) & $\mathrm{I}$ \\
Pulmonary cyst & $\mathrm{I}$ \\
\hline
\end{tabular}

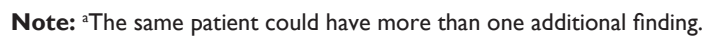

Abbreviations: CT, computed tomography; LDCT, low-dose computed tomography. radiography. ${ }^{6,11}$ However, because there are radiation risks in using LDCT lung cancer screening, it should be performed only on current or former tobacco smokers. ${ }^{6,11}$ In this study, nodular changes were observed in as many as 19 cases (five apparently healthy current or former smokers and 14 COPD patients) (Table 2), possibly causing anxiety for patients and problems for the clinicians requested to follow-up. Nine patients (13\%) needed further follow-up and, of these, three patients $(4.3 \%)$ were found to have lung cancer. V/P SPECT might provide a more comprehensive functional assessment that can guide decisions about surgery (Figure 2) in patients finally diagnosed with cancer, as suggested by Yuan et al. ${ }^{12}$

An interesting finding in this study was that 13 out of 14 apparently healthy smokers showed various degrees of obstructive airway changes. V/P SPECT has the possibility to assess how ventilation and perfusion are affected and to detect early functional changes in small airways. These findings are in agreement with those of Rodríguez-Roisin et al, who measured $\mathrm{V} / \mathrm{P}$ ratios in the lung using the multiple inert gas elimination technique and showed high sensitivity to COPD pathologies. ${ }^{13}$ A recent paper by Jobse et al further affirmed that V/P mismatching progressively increased during cigarette smoke exposure in mice. ${ }^{14}$ While spirometry offers a simple test for COPD, it only provides a global measure, is insensitive to early disease, and cannot explore the heterogeneity of COPD. V/P SPECT greatly augments our understanding of the disease and offers the possibility to see how various pathologies affect gas exchange. V/P SPECT has the ability to visualize early functional impairment leading to COPD that may not be obvious, even at a higher resolution, in anatomical imaging systems such as CT. ${ }^{14}$

The primary indication for the use of V/P SPECT is PE. ${ }^{2,9}$ Previous studies have shown that V/ P SPECT alone has less than $4 \%$ nondiagnostic examinations, as well as high sensitivity and specificity. ${ }^{15-21}$ Because of the high sensitivity and specificity of V/P SPECT alone, it is not generally recommended to use a hybrid system for PE diagnosis, as argued by some authors. ${ }^{7}$ As this study shows, some patient groups may, however, benefit from the additional anatomical information that LDCT provides, especially patients with an elevated risk of lung malignancy. Therefore, it might be appropriate to recommend the use of hybrid imaging among patients with COPD but also among apparently healthy long-term smokers. This needs to be studied further in larger studies. In the light of the increasing awareness regarding the overuse of $\mathrm{CT}$, as noted in several publications in recent years, ${ }^{22-24}$ it is, however, not 

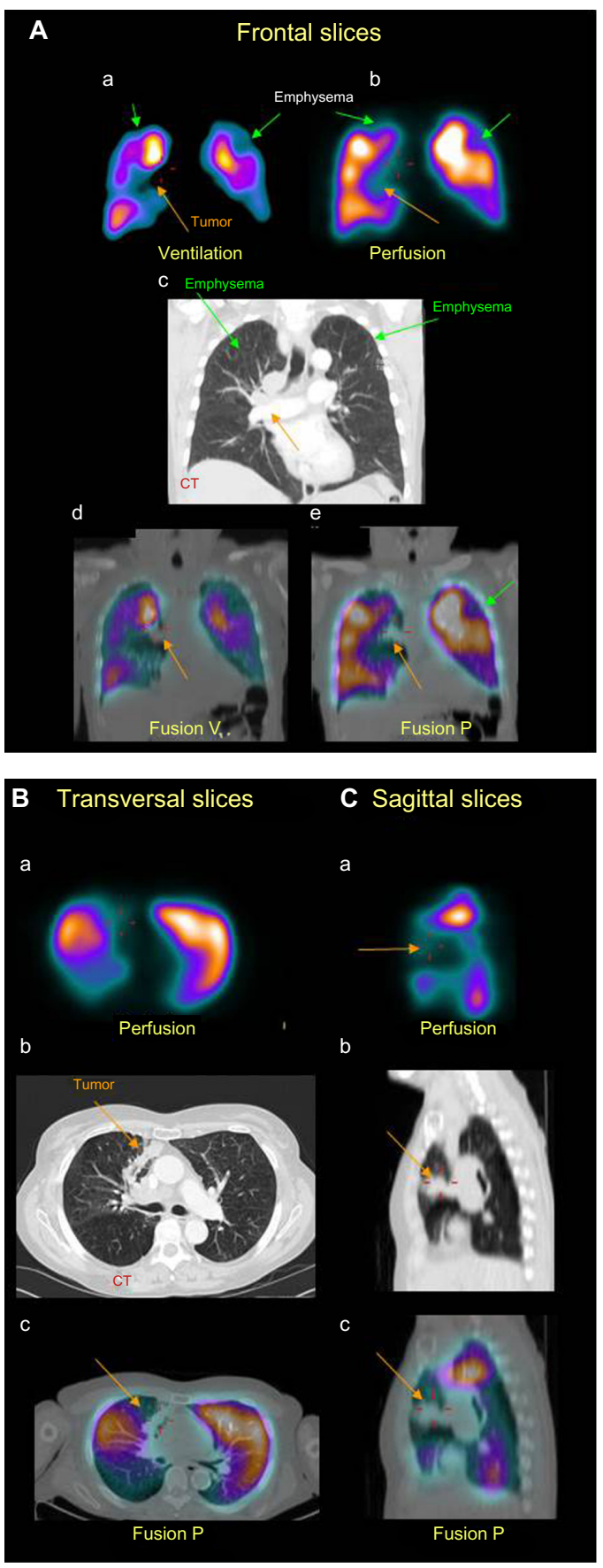

Figure 2 Patient with COPD and tumor.

Notes: (A) Coronal slices display uneven distribution of ventilation (a) with deposition of aerosol (Technegas) in central and intermediate airways that is typical for airway obstruction and COPD. Perfusion (b) follows the ventilation pattern. (c) Matched ventilation and perfusion defects are observed in both upper lobes (green arrows) and to the right in the mediastinum (orange arrows). In the corresponding frontal CT slice (c), emphysema is observed in both upper lobes (green arrows), as is a tumor in the mediastinum (orange arrow). Fusion images of CT and ventilation SPECT (d) and CT and perfusion SPECT (e) are shown. Orange arrows indicate the tumor and the perfusion defect it gives rise to. Green arrows indicate emphysema. (B) Transverse slice of perfusion (a), with corresponding CT slice (b). (tumor indicated with orange arrow) A large perfusion defect (orange arrow) is caused by the tumor, which can be clearly seen in the fusion image (c). (C) Sagittal slices show an extensive area with absent perfusion (orange arrow) (a) caused by the tumor (orange arrows) seen on CT (b) and on the fusion image (c).

Abbreviations: COPD, chronic obstructive pulmonary disease; $\mathrm{CT}$, computed tomography; SPECT, single-photon emission computed tomography. acceptable to advocate the extra radiation delivered from CT for every patient with suspected PE as the prevalence of $\mathrm{PE}$ is as low as $10 \%-30 \%$ in most study populations. ${ }^{15,25,26}$ A better routine for selecting patients who may benefit from hybrid imaging is therefore needed to solve the problem of false positives in tumor diagnosis.

It is known that COPD patients often have clinically significant pulmonary vascular changes. ${ }^{27}$ In this study, as well as in an earlier study by our group, ${ }^{3}$ it was shown that vascular defects exist both in apparently healthy smokers and COPD patients. The effects of the vascular changes can be shown with V/P SPECT. Estépar et al also found distal pruning of the pulmonary blood vessels using CT among smokers. ${ }^{28}$

We have many diagnostic imaging modalities at our disposal today. The question is how best to use them for the benefit of our patients. To decide on the most appropriate diagnostic modality, it is most important to focus on the clinical question. When hybrid imaging is used for the lungs, this study shows that, at least among smokers and patients with COPD, the LDCT images need to be evaluated with malignancy in mind. It is not adequate to regard the LDCT images only as a way to localize physiological findings. This is a new challenge for nuclear medicine physicians.

\section{Limitations}

The number of patients in this study is limited, and larger studies will be needed. The finding that LDCT identifies previously undetected lung cancer in this group of patients is in line with previous studies. ${ }^{6,11}$ This is, however, to our knowledge, the first study that shows this in patients examined with a hybrid system. SPECT/CT acquisition of the chest constitutes a challenge due to respiratory movements, which can cause image artifacts and thus decrease diagnostic accuracy. All images were, however, regarded as diagnostic by the interpreters. To reduce the risk of artifacts, the CT was not used for attenuation correction.

\section{Conclusion}

In patients with COPD and apparently healthy long-time smokers, V/P SPECT/CT hybrid imaging appears to have added value compared to V/P SPECT alone. The additional value of LDCT in this group was primarily the ability to identify patients with suspected lung malignancy and to more clearly identify and categorize emphysema. V/P SPECT can visualize complications of COPD, such as PE and decompensated heart failure, that cannot be seen with LDCT. 


\section{Acknowledgments}

This study was funded in part by Region of Scania (ALF) and the Swedish Heart-Lung Foundation. We want to thank the staff at the Department of Clinical Physiology and Nuclear Medicine, Skåne University Hospital, Lund, Sweden.

\section{Disclosure}

The authors report no conflicts of interest in this work.

\section{References}

1. Vestbo J, Hurd SS, Agustí AG, et al. Global strategy for the diagnosis, management, and prevention of chronic obstructive pulmonary disease: GOLD executive summary. Am J Respir Crit Care Med. 2013; 187(4):347-365.

2. Bajc M, Neilly JB, Miniati M, Schuemichen C, Meignan M, Jonson B. EANM guidelines for ventilation/perfusion scintigraphy: Part 2. Algorithms and clinical considerations for diagnosis of pulmonary emboli with V/P(SPECT) and MDCT. Eur J Nucl Med Mol Imaging. 2009;36(9):1528-1538.

3. Jögi J, Ekberg M, Jonson B, Bozovic G, Bajc M. Ventilation/perfusion SPECT in chronic obstructive pulmonary disease: an evaluation by reference to symptoms, spirometric lung function and emphysema, as assessed with HRCT. Eur J Nucl Med Mol Imaging. 2011;38(7): 1344-1352.

4. Bajc M. Potential of hybrid V/P SPECT - low-dose CT in lung diagnostics. Breathe. 2012;9(1):49-60.

5. Kashyap R, Dondi M, Paez D, Mariani G. Hybrid imaging worldwide-challenges and opportunities for the developing world: a report of a Technical Meeting organized by IAEA. Semin Nucl Med. 2013;43(3):208-223.

6. Nanavaty P, Alvarez MS, Alberts WM. Lung cancer screening: advantages, controversies, and applications. Cancer Control. 2014; 21(1):9-14.

7. Gutte H, Mortensen J, Jensen CV, et al. Detection of pulmonary embolism with combined ventilation-perfusion SPECT and low-dose CT: head-to-head comparison with multidetector CT angiography. $\mathrm{J} \mathrm{Nucl}$ Med. 2009;50:1987-1992.

8. [No authors listed]. Standardization of Spirometry, 1994 Update. American Thoracic Society. Am J Respir Crit Care Med. 1995;152(3): 1107-1136.

9. Bajc M, Neilly JB, Miniati M, Schuemichen C, Meignan M, Jonson B; EANM Committee EANM guidelines for ventilation/ perfusion scintigraphy: Part 1. Pulmonary imaging with ventilation/ perfusion single photon emission tomography. Eur J Nucl Med Mol Imaging. 2009;36(8):1356-1370.

10. Jögi J, Palmer J, Jonson B, Bajc M. Heart failure diagnostics based on ventilation/perfusion single photon emission computed tomography pattern and quantitative perfusion gradients. Nucl Med Commun. 2008; 29(8):666-673.

11. Furlow B. Low-dose computed tomography lung cancer screening. Radiol Technol. 2014;85(3):297CT-314CT; quiz 315CT-318CT.
12. Yuan ST, Frey KA, Gross MD, et al. Changes in global function and regional ventilation and perfusion on SPECT during the course of radiotherapy in patients with non-small-cell lung cancer. Int J Radiat Oncol Biol Phys. 2012;82(4):e631-e638.

13. Rodríguez-Roisin R, Drakulovic M, Rodríguez DA, Roca J, Barberà JA, Wagner PD. Ventilation-perfusion imbalance and chronic obstructive pulmonary disease staging severity. J Appl Physiol (1985). 2009; 106(6):1902-1908.

14. Jobse BN, Rhem RG, McCurry CA, Wang IQ, Labiris NR. Imaging lung function in mice using SPECT/CT and per-voxel analysis. PLoS One. 2012;7(8):e42187.

15. Bajc M, Olsson B, Palmer J, Jonson B. Ventilation/perfusion SPECT for diagnostics of pulmonary embolism in clinical practice. $J$ Intern Med. 2008;264(4):379-387.

16. Leblanc M, Leveillée F, Turcotte E. Prospective evaluation of the negative predictive value of V/Q SPECT using $99 \mathrm{mTc}$-Technegas. Nucl Med Commun. 2007;28(8):667-672.

17. Lemb M, Pohlabeln H. Pulmonary thromboembolism: a retrospective study on the examination of 991 patients by ventilation/perfusion SPECT using Technegas. Nuklearmedizin. 2001;40(6):179-186.

18. Elf JE, Jögi J, Bajc M. Home treatment of patients with small to medium sized acute pulmonary embolism. J Thromb Thrombolysis. Epub June 19, 2014.

19. Grüning T, Drake BE, Farrell SL, Nokes T. Three-year clinical experience with VQ SPECT for diagnosing pulmonary embolism: diagnostic performance. Clin Imaging. Epub April 23, 2014.

20. Kan Y, Yuan L, Meeks JK, Li C, Liu W, Yang J. The accuracy of V/Q SPECT in the diagnosis of pulmonary embolism: a meta-analysis. Acta Radiol. Epub June 10, 2014.

21. Quirce R, Ibáñez-Bravo S, Jiménez-Bonilla J, et al. Contribution of V/Q SPECT to planar scintigraphy in the diagnosis of pulmonary embolism. Rev Esp Med Nucl Imagen Mol. 2014;33(3):153-158.

22. Coco AS, O'Gurek DT. Increased emergency department computed tomography use for common chest symptoms without clear patient benefits. J Am Board Fam Med. 2012;25(1):33-41.

23. Einstein AJ, Henzlova MJ, Rajagopalan S. Estimating risk of cancer associated with radiation exposure from 64-slice computed tomography coronary angiography. JAMA. 2007;298(3):317-323.

24. Roche LM, Niu X, Pawlish KS, Henry KA. Thyroid cancer incidence in New Jersey: time trend, birth cohort and socioeconomic status analysis (1979-2006). J Environ Public Health. 2011;2011:850105.

25. Mamlouk MD, vanSonnenberg E, Gosalia R, et al. Pulmonary embolism at CT angiography: implications for appropriateness, cost, and radiation exposure in 2003 patients. Radiology. 2010;256(2):625-632.

26. Stein PD, Fowler SE, Goodman LR, et al; PIOPED II Investigators. Multidetector computed tomography for acute pulmonary embolism. N Engl J Med. 2006;354(22):2317-2327.

27. Minai OA, Chaouat A, Adnot S. Pulmonary hypertension in COPD: epidemiology, significance, and management: pulmonary vascular disease: the global perspective. Chest. 2010;137(Suppl 6):39S-51S.

28. Estépar RS, Kinney GL, Black-Shinn JL, et al; COPD Gene Study. Computed tomographic measures of pulmonary vascular morphology in smokers and their clinical implications. Am J Respir Crit Care Med. 2013;188(2):231-239.
International Journal of COPD

\section{Publish your work in this journal}

The International Journal of COPD is an international, peer-reviewed journal of therapeutics and pharmacology focusing on concise rapid reporting of clinical studies and reviews in COPD. Special focus is given to the pathophysiological processes underlying the disease, intervention programs, patient focused education, and self management protocols.

\section{Dovepress}

This journal is indexed on PubMed Central, MedLine and CAS. The manuscript management system is completely online and includes a very quick and fair peer-review system, which is all easy to use. Visit http://www.dovepress.com/testimonials.php to read real quotes from published authors. 